\title{
Performance Analysis of a 2-D Time-wavelength OCDMA Wavelength-Aware Receiver with Beat Noise
}

\author{
Taher Bazan, David Harle, Ivan Andonovic \\ University of Strathclyde, 204 George Street, G1 1XW Glasgow, Scottland, United Kingdom \\ Tel: (44) 548 2082, Fax: (44) 5524968 ,e-mail:taher.bazan@strath.ac.uk
}

\begin{abstract}
The effect of beat noise on two-dimensional time-wavelength optical code-division multiple-access systems utilising wavelength-aware receivers is examined. A derivation of a general formula for the bit error probability taking into consideration multiple access interference (MAI) and other noise sources is given. In addition, a comparison between the system performance of such a receiver and the traditional configuration is presented. Studies to date that have focused only on the MAI limited case showed that the wavelength-aware configuration yields a better performance when compared to the traditional receiver. When beat noise is considered, the numerical results reveal that the performance of wavelength-aware receiver is very sensitive to beat noise and is not superior over the traditional receiver.
\end{abstract}

\section{INTRODUCTION}

Two-dimensional (2-D) time-wavelength (TW) optical code-division multiple-access (OCDMA) systems have attracted much attention since they offer a larger code size, superior auto-and cross-correlation properties and higher bandwidth efficiency [1-2]. Low co-channel interference is inherent to 2-D codes since interfering pulses must overlap in both time and wavelength. Recently, a wavelength-aware receiver (WAR) [3] was introduced as an effective way to enhancing the system performance over the traditional configuration [4], doubling the spectral efficiency. However, such a study has only been carried out under the multiple access interference (MAI) limited case.

One of the limiting factors with TW schemes and indeed other OCDMA coding approaches, is beat noise (BN) [5-6] which occurs when different optical signals - the main signal and crosstalk term(s) - at nominally the same frequencies are incident simultaneously on a photodetector. Due to the action of the square law detector, the photocurrent owing to $\mathrm{BN}$ is much greater than that attributed to the incident optical power of the crosstalk signal, producing severe system performance degradations.

In this paper, continuing on previous work [3], the behaviour of a 2-D TW OCDMA system employing wavelength-aware receivers taking into account beat noise is examined. The system model and assumptions for a 2-D TW OCDMA scheme utilising coherent laser sources are given in Section 2. In Section 3, a general formula for the error probability of the system is introduced. Within Section 4, results and discussions are presented and finally, conclusions are drawn in Section 5.

\section{SYSTEM DESCRIPTION}

\subsection{System model}

The 2-D OCDMA system under consideration consists of multiple transmitters and receivers within a star configuration. At the transmitter, the optical source is modulated according to the user data and the output is encoded in two dimensions. In normal multi-user environments, the encoded signals of the intended user and other users are simultaneously incident at the receiver. The following assumptions are applied in the analysis [5-6]:

- all signals are on-off keyed (OOK) and in a non-return to zero (NRZ) format.

- code chips are generated using arrays of single mode lasers or tunable lasers.

- chirp-free external modulation and infinite modulation depth.

- chip synchronous case, resulting in an upper bound in relation to the asynchronous system performance. This does not infer that the system operation is synchronous; chip asynchronicity is assumed.

- all interferers are of the same intensity at the receiver.

- data and interferer pulses are of the same polarization state (worst case).

- the frequency separation between chips at different wavelengths exceeds the receiver electrical bandwidth. Also, the frequency between interferer chips at different wavelengths exceeds the receiver's electrical bandwidth. Thus, the model focuses only the beat noise caused by the overlap of identical wavelengths.

\subsection{Principle of operation}

Figure 1 shows the block diagram of the WAR, 
comprising an optical 2-D TW decoder followed by arrays of photodetectors and electronic thresholding elements, the outputs of which are connected to an electronic AND gate. The number of photodetectors is equivalent to the code weight (active chips). Each branch, independently, decides on the existence of each active chip within the intended code. A bit " 1 " is detected if at least one pulse is present at each branch and the absence of a pulse is sufficient to declare a " 0 " bit. It is clear that the WAR requires more hardware than the traditional receiver since the latter comprises a decoder, single PD and ET.

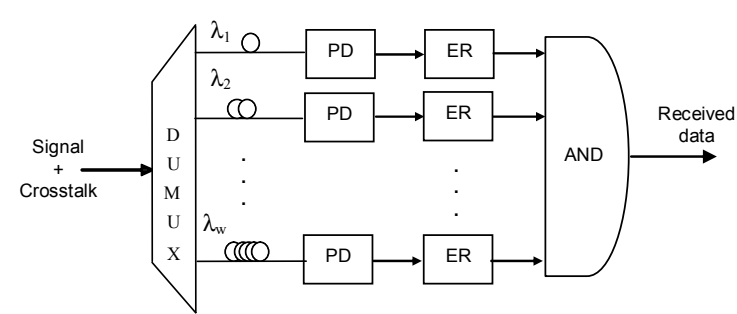

Figure 1. Wavelength aware receiver architecture. $P D$ denotes the photodetector and ET is an electronic threshold element.

\section{PERFORMANCE EVALUATION}

In this section, the system performance in terms of bit error rate (BER) is derived taking into account the dual effects of MAI and beat noise. The main difference between beat noise and MAI is that BN affects both bits " 1 " and " 0 " with additional levels of beat noise expected when transmitting bit "1". The error contributions owing to both data bits must be evaluated. Consider the case of transmitting a desired data bit "0"; an error occurs when the receiver decides a bit "1" due to detection of a mark in all " 0 " positions. Such a case is conditioned by the distribution of interference patterns. Assume that " $\mathrm{j}$ " interferers produce hits with the desired code and every interferering user contributes only one pulse. Therefore, all the possible combinations of interference patterns arising from simultaneous users can be formulated by defining $F_{j}$ viz. $F_{j}$ contains a set of vectors (or basic interference patterns) representing all interfering users. Note that each basic interference pattern contains all the permutation of such a vector. The number of vector permutations and the corresponding multinomial probability distribution (MPD) for a specific value of " $j$ " can be expressed respectively as follows [2]:

$$
\begin{gathered}
N D P(\bar{\alpha})=\frac{w !}{\prod_{i} R\left(\alpha_{i}\right)} \\
\operatorname{MPD}\left(\bar{\alpha} ; F_{j}\right)=\frac{j !}{w^{j} \cdot \prod_{d=1}^{w}\left(\alpha_{d} !\right)}
\end{gathered}
$$

where $R\left(\alpha_{i}\right)$ is the repetition of element $\alpha_{\mathrm{i}}$ in the vector and $w$ is the code weight. Amongst $F_{j}$, a sub-set of vectors or patterns will create errors, denoted by a set $G_{j}$. When transmitting a bit " 0 ", $G_{j}$ contains all non-zero vectors affecting all " 0 " chip positions. The probability of error due to transmitting bit " 0 ", $P(E \mid 0)$, can be conditioned on the following events. " $i$ " users among the (K-1) probable interferers may transmit a data bit " 1 " with probability equal to " $1 / 2$ ". Among these "i" users, there exist " $\mathrm{j}$ " users that may have a hit at the autocorrelation peak of the desired user viz. crosstalk. The average probability of a hit follows a binomial distribution and is equal to " $h$ av". Such a parameter is dependent on the 2-D TW code utilised. Therefore, the bit error probability for the intended user on transmission of bit " 0 " is given by:

$$
P(E \mid 0)=\frac{1}{2} \sum_{i=w}^{K-1}\left(\begin{array}{c}
K-1 \\
i
\end{array}\right) \cdot 2^{-(K-1)} \cdot \sum_{j=w}^{i}\left(\begin{array}{l}
i \\
j
\end{array}\right) \cdot h_{a v}^{j} \cdot\left(1-h_{a v}\right)^{i-j} \cdot \sum_{\alpha_{d} \in G_{j}} P_{E}\left(\bar{\alpha}_{d}\right)
$$

$P_{E}(\bar{\alpha})$ is the probability of error given that the appropriate pattern is received. The minimum number of users required to produce an error, for the case of transmitting bit " 0 ", is equal to the code weight. Consequently, the summations in Equation (3) starts with $i=w$. The probability, $P_{E}(\bar{\alpha})$, can be computed as follows:

$$
P_{E}\left(\bar{\alpha}_{d}\right)=\prod_{m=1}^{w} N D P\left(\alpha_{d}\right) \cdot M P D\left(\alpha_{d}\right) \cdot Q\left(\frac{p_{d} \cdot D-\alpha_{m} \cdot P_{c}}{\sqrt{\sigma_{t h}^{2}+\sigma_{s h_{0}}^{2}+\sigma_{R I N_{0}}^{2}+\sigma_{B N_{0}}^{2}}}\right)
$$

where $P_{d}$ and $P_{c}$ are the instantaneous optical chip power of the data signal and crosstalk respectively and $\mathrm{D}$ is the threshold level. The variance of thermal, shot, relative intensity noise (RIN) and beat noise can be expressed respectively by:

$$
\begin{gathered}
\sigma_{t h}^{2}=\frac{4 \cdot K_{B} \cdot T \cdot B_{e}}{R_{L}}, \sigma_{s h_{0}}^{2}=2 \cdot q \cdot I \cdot B_{e}, \sigma_{R I N_{0}}^{2}=R I N \cdot I^{2} \cdot B_{e}, \sigma_{B N_{0}}^{2}=2 \cdot P_{c} \cdot P_{c} \cdot\left(\begin{array}{c}
\alpha_{m} \\
2
\end{array}\right) \\
I=\alpha_{m} \cdot P_{c}
\end{gathered}
$$


where $K_{B}$ is the Boltzman's constant, $T$ is the absolute temperature in degree Kelvin, $R_{L}$ is the receiver load resistance, $B_{e}$ is the receiver electrical bandwidth, $q$ is the electron charge, and RIN is the relative intensity noise parameter $(\mathrm{dB} / \mathrm{Hz})$. The parameter $\alpha_{m}$ representing the number of overlaps at the specific colored chip position is $\sum_{m=1}^{w} \alpha_{m}=j$. Combining equations (3) and (4), the probability of error due to the transmission of bit " 0 " can be written as follow:

$$
P(E \mid 0)=\frac{1}{2} \sum_{i=w}^{K-1} \frac{\left(\begin{array}{c}
K-1 \\
i
\end{array}\right)}{2^{(K-1)}} \cdot \sum_{j=w}^{i}\left(\begin{array}{l}
i \\
j
\end{array}\right) \cdot h_{a v}^{j} \cdot(1-h)^{i-j} \cdot \sum_{\alpha_{d} \in G_{j}} \prod_{m=1}^{w} N D P\left(\alpha_{d}\right) \cdot M P D\left(\alpha_{d}\right) \cdot Q\left(\frac{p_{d} \cdot D-\alpha_{m} \cdot P_{c}}{\sigma_{t o t_{0}}}\right)
$$

Now consider the case of transmitting a data bit " 1 "; an error occurs when at least one active chip, i.e. wavelength, is detected in the time slot of bit " 0 ". In this case, the damage vectors are both zero and non-zero since a wrong decision at any chip position will produce an error. Therefore, $F_{j}=G_{j}$ and consequently, more damage patterns producing errors are likely. Now the minimum number of users required to produce an error is 1 instead of $w$. Following the previous equations, the probability of error resulting from the transmission of bit " 1 " can be written as:

$$
P(E \mid 1)=\frac{1}{2} \sum_{i=1}^{K-1} \frac{\left(\begin{array}{c}
K-1 \\
i
\end{array}\right)}{2^{(K-1)}} \cdot \sum_{j=1}^{i}\left(\begin{array}{l}
i \\
j
\end{array}\right) \cdot h_{a v}^{j} \cdot\left(1-h_{a v}\right)^{i-j} \cdot \sum_{\alpha_{d} \in F_{j}} \sum_{m=1}^{w} N D P\left(\bar{\alpha}_{d}\right) \cdot M P D\left(\bar{\alpha}_{d}\right) \cdot Q\left(\frac{P_{d}+\alpha_{m} \cdot P_{c}-P_{d} \cdot D}{\sqrt{\sigma_{t h}^{2}+\sigma_{s h_{1}}^{2}+\sigma_{R I N_{1}}^{2}+\sigma_{B N_{1}}^{2}}}\right)
$$

The noise variance in equation (7) is similar to (5) but the average current is $I=P_{d}+\alpha_{m} . P_{c}$ and the variance of beat noise is given by:

$$
\sigma_{B N_{1}}^{2}=2 \cdot \alpha_{m} \cdot P_{d} \cdot P_{c}+2 \cdot P_{c} \cdot P_{c} \cdot\left(\begin{array}{c}
\alpha_{m} \\
2
\end{array}\right)
$$

Thus, the total bit error rate for wavelength-aware receivers is the summation of equations (6) and (7). For MAI limited case, the probability of error can be expressed as:

$$
P_{E}=\frac{1}{2} \sum_{i=w}^{K-1}\left(\begin{array}{c}
K-1 \\
i
\end{array}\right) \cdot 2^{(K-1)} \cdot \sum_{j=w}^{i}\left(\begin{array}{l}
i \\
j
\end{array}\right) \cdot h_{a v}^{j} \cdot(1-h)^{i-j} \cdot \sum_{\bar{\alpha}_{d} \in G_{j}} \prod_{m=1}^{w} N D P\left(\bar{\alpha}_{d}\right) \cdot \operatorname{MPD}\left(\bar{\alpha}_{d}\right)
$$

The analysis framework developed previously can be best illustrated by the following example. Let the number of interferers be $j=6$ and the code weight $w=3$. Thus, all the possible combinations of interference patterns are: $F_{6}=\{(6,0,0),(5,1,0),(4,2,0),(4,1,1),(3,3,0),(3,2,1),(2,2,2)$, and all vectors permutations $\}$. Thus, the total number of vectors in $F_{6}=28$ and all those vectors may affect the decision of bit "1". On the other hand, amongst $F_{j}$, only the non-zero vectors containing any non-zero element can produce an error for bit " 0 ". Thus, $G_{6}=$ $\{(4,1,1),(3,2,1),(2,2,2)\}$. According to equation $(1)$, the $N D P$ of the basic vectors are $N D P(4,1,1)=3 ! /(1 ! .2 !)=3$, $\operatorname{NDP}(3,2,1)=3 ! /(1 ! .1 ! .1 !)=6$, and $\operatorname{NDP}(2,2,2)=3 ! / 3 !=1$; the total number of non-zero vectors including their permutations are 10. The corresponding multinomial probability distributions can be evaluated according to equation (2) such that: $\operatorname{MPD}(4,1,1)=6 ! /\left(3^{6} .4 ! .1 ! .1 !\right)=0.0411$ and so on.

\section{RESULTS AND DISCUSSIONS}

System performance is calculated assuming that a bit rate $100 \mathrm{MHz}, R_{L}=1 \mathrm{k} \Omega, \quad \mathrm{RIN}=-150 \mathrm{~dB} / \mathrm{Hz}$, $P_{d}=P c=1.42 \mathrm{~mW}$ and employing a 2-D carrier-hopping prime code (CHPC) of weight $w=7$ and length $N=101$ [2]. For CHPC, $h_{a v}=w / N$. The bit error rate (BER) is plotted as a function of number of simultaneous users with and without $\mathrm{BN}$ and compared to the traditional receiver (Fig. 2). It is clear that the results emphasize the superior performance of WAR over the traditional receiver for the MAI limited case is evident. However, with $\mathrm{BN}$, the performance of WAR is worse than the traditional receiver; $2 \mathrm{~dB}$ at 5 users. Furthermore, as the number of users' increases this penalty becomes smaller. The main source of the limits in WAR or the traditional receiver is the error due to receiving a bit " 1 ". For WAR, if any chip is corrupted by BN according to a specific interference pattern, then the decision is false, independent of other chips. The traditional receiver decides whether the receiving bit is " 1 " or not based on the accumulative effect of BN on all chips. So, for the same interference pattern, if one chip is affected, BN does not necessarily affect the overall decision. The situation for the MAI case is reversed where the only limiting factor is due to transmission of bit " 0 ". If all chips in WAR are simultaneously corrupted, an error occurs. This probability is very low when compared to the traditional receiver where, if sufficiently high levels of beat noise occur at any chip, then an error may result. However, increasing 
the code length results in slightly improved performance since the probability of a hit is reduced whereas the performance is worsens with increasing code weight (Fig. 3).

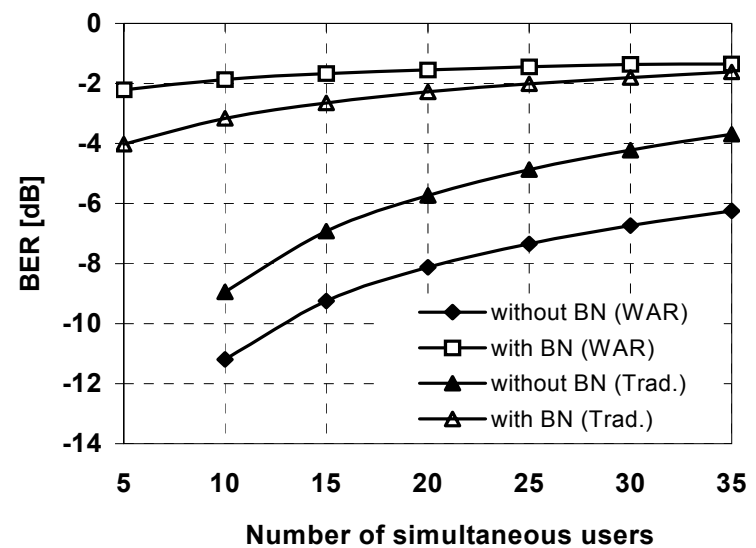

Figure 2. BER versus number of simultaneous users with/without BN for WAR and traditional receiver.

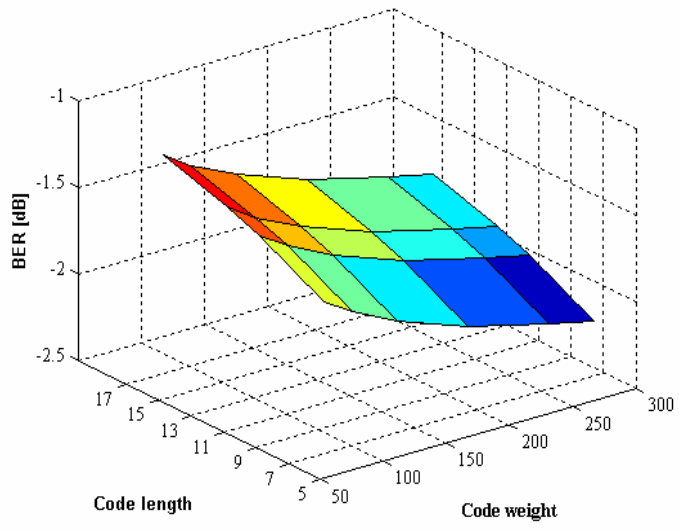

Figure 3. BER versus (weight, length).

\section{CONCLUSIONS}

A performance analysis of a 2-D time-wavelength OCDMA system utilising coherent light sources and a wavelength-aware configuration at the receiver has been presented. The analysis includes major system noise sources but focuses on beat noise and multiple access interference. Results show that the detrimental effect of beat noise on such a system and, contrary to the MAI limited case, the performance of WAR under beat noise yields no improvement when compared to the traditional receiver. Although the WAR geometry appears to offer improved performance, the analysis show that when all form of interference is considered, and especially beat noise, the performance of WAR degrades significantly. This result has implications for the use of such a receiver.

\section{REFERENCES}

[1] L. Tančevski, I. Andonovic: Wavelength hopping/time spreading code division multiple access systems, Electron. Lett., vol. 30, pp. 1388-1390, Aug. 1994.

[2] G.-C. Yang, W. Kwong: Prime Codes With Applications to CDMA Optical and Wireless Networks, Boston, MA: Artech House, 2002.

[3] V. Baby, C.S. Bress, I. Glesk, P. Prucnal: Wavelength aware receiver for enhancement 2-D CDMA system performance, Electron. Lett., vol. 40, pp. 385-386, Mar. 2004.

[4] J. Salehi, C. Brackett: Code division multiple -access techniques in optical fiber networks- Part II: System performance analysis, IEEE Trans. Comm., vol.37, pp. 834-842, Aug. 1989.

[5] L. Tančevski and L.A. Rusch, " Impact of beat noise on the performance of 2-D optical CDMA systems, IEEE Comm. Lett., vol. 4, no. 8, pp.264-266, Aug. 2000.

[6] T. Bazan, D. Harle, and I. Andonovic, M. Meenakshi: Effect of beat noise on the performance of 2-D timespreading wavelength-hopping optical code-division multiple-access systems, J. Optical Networking, vol. 4, pp. 121-129, Mar. 2005. 